\title{
LE PROBLEMATICHE SOCIO-GIURIDICHE CONNESSE ALL'IMMIGRAZIONE ISLAMICA IN EUROPA CON PARTICOLARE RIGUARDO ALLA SITUAZIONE ITALIANA*
}

\author{
Carlo De Angelo
}

\begin{abstract}
NAPLES
This study deals with the migration flows from Islamic countries, or countries with large Islamic populations, to Europe. Particular attention is paid to the factors that explain these flows from the 1950s to the 1970s. After the restrictive policies or closure introduced in the 1970s, migration shows a clear trend towards permanent settling and takes on new and more defined characteristics. The social-juridical consequences related to these transformations are examined here. The second part of the study describes differences between the Italian and the more general European situation. Sizeable Islamic presence in Italy is a relatively new phenomenon, dating back largely to the 1980s. Unlike elsewhere in Europe, Islam has established itself very rapidly. The whole typology of Islamic institutionalized presence characteristic of other European countries is already visible. As elsewhere, the Islamic community-particularly through the action of the UCOII, the most representative umbrella organization -is trying to reach an intesa (agreement) with the government to obtain a status comparable with that of other religious minorities. However, as of this writing, the process has not yet officially begun. This can be ascribed in particular to the problem of determining which Islamic body should be taken as the legitimate representative of the Muslim communities. With reference to other juridical problems (mosques, haläl food, cemeteries, hiğäb, marriage, etc.) the paper defends the position that a sustainable immigration policy should be able to reflect the interests of both immigrants and native inhabitants. The most effective strategy, it would seem, can be worked out within a cross-cultural perspective that recognizes that confrontation and dialogue are possible, indeed necessary, among cultures sharing a common core of values and principles, that is to say, a common acceptance of universal human rights.
\end{abstract}

\footnotetext{
* Questo articolo costituisce una sintesi della mia tesi di laurea in Diritto musulmano e dei paesi islamici, intitolata "Immigrazione islamica e ordinamento giuridico italiano: conflitti ipotizzabili e soluzioni possibili", discussa presso la Facoltà di Scienze Politiche dell'Istituto Universitario Orientale di Napoli (a.a. 1999-2000), presentata dal Prof. Agostino Cilardo.
} 


\section{L'EVOLUZIONE DELL'IMMIGRAZIONE ISLAMICA IN EUROPA E DEL RELATIVO PROCESSO DI VISIBILITÀ}

La presenza di circa dieci milioni di musulmani residenti nell'Unione Europea si è costituita tramite consistenti flussi migratori, che negli ultimi cinquant'anni si sono diretti in Europa, avendo per lo più come provenienza paesi africani ed asiatici in cui la religione islamica è del tutto prevalente o almeno molto diffusa. Con la stabilizzazione degli immigrati nei vari paesi europei si è poi verificata la ricostituzione e la nuova costituzione di nuclei familiari che hanno determinato la comparsa delle nuove generazioni, spesso in possesso della cittadinanza degli Stati di accoglienza. L'insieme di queste due componenti, ossia i residenti stranieri originari di paesi islamici a cui va aggiunta la seconda e terza generazione di origine immigrata, costituisce la presenza islamica in Europa occidentale. ${ }^{1}$

Il processo migratorio da cui trae origine questa presenza si è andato sviluppando attraverso l'evolversi di quattro cicli migratori: ${ }^{2}$ dal 1945 alla fine degli anni sessanta; dalla fine degli anni sessanta al 1973; dal 1973 al 1980; dal 1980 ad oggi.

I primi due cicli, cioè quelli che hanno avuto luogo fra il 1950 e il 1970, possono esser trattati congiuntamente dal momento che in entrambi i casi i flussi provenienti dai paesi islamici erano inseriti in un processo di trasferimento di manodopera che si verificava prevalentemente secondo accordi tra Stati o istituzioni di primo piano, ${ }^{3}$ un trasferimento che risultava funzionale sia ai paesi di provenienza che a quelli di destinazione. ${ }^{4}$ Questi ultimi, infatti, furono spinti dalla carenza di risorse umane indispensabili alla ricostruzione postbellica (anni '50-'60, primo ciclo migratorio) nonché dal successivo lungo periodo di crescita eco-

${ }^{1}$ Cf. Pacini, A., «I musulmani in Italia. Dinamiche organizzative e processi di interazione con la società e le istituzioni italiane», in Ferrari, S. (a cura di), Musulmani in Italia. La condizione giuridica delle comunità islamiche, Il Mulino, Bologna, 2000, p. 21.

${ }^{2}$ Per ciclo migratorio s'intende "il processo attraverso il quale popolazioni appartenenti ad uno spazio economico periferico entrano, si stabiliscono e s'insediano nello spazio di uno Stato-nazione che appartiene ai poli centrali dell'economia capitalistica"; cf. Dassetto, F., Bastanier, A., Europa: nuova frontiera dell'Islam, Edizioni Lavoro, Roma, 1991, p. 251.

${ }^{3}$ Cf. Collinson, S., Le migrazioni internazionali e l'Europa, Il Mulino, Bologna, 1994, pp. 35-40.

${ }^{4}$ Cf. Pacini, A., «I musulmani in Italia», p. 23. 
nomica che interessò i paesi dell'Europa nordoccidentale (anni '60-'70, secondo ciclo migratorio), ad una progressiva espansione dei rispettivi mercati del lavoro, così da incorporare lavoratori provenienti non solo da paesi europei meno sviluppati (Italia, Spagna, Portogallo, Irlanda) ma anche, e poi successivamente in maniera esclusiva, da paesi non europei, comprese le colonie e le ex colonie. ${ }^{5}$ Dal canto loro, queste ultime, incoraggiati dall'elevata domanda di manodopera nell'Europa nordoccidentale, cominciarono ad attuare politiche atte a promuovere l'esportazione di forza lavoro, individuando così nell'emigrazione la strategia più adatta a risolvere quei problemi connessi all'occupazione e alla bilancia dei pagamenti ${ }^{6}$ che erano considerati di impedimento allo sviluppo economico.

Una delle caratteristiche di questi primi due cicli migratori è quindi rintracciabile nel fatto di essersi sviluppati perpetuando quei legami di familiarità instaurati tra alcuni paesi durante il periodo coloniale. Anche se questa non è stata una costante assoluta, tuttavia gli emigrati hanno spesso scelto come meta i paesi con cui avevano avuto relazioni coloniali e che risultavano quindi più familiari; ${ }^{7}$ questo spiega perché tutti gli Stati europei che hanno conosciuto un'immigrazione più antica, abbiano oggi popolazioni immigrate caratterizzate dalla netta preponderanza di una o due nazionalità specifiche. ${ }^{8}$

I musulmani, che nel ventennio 1950-1970 si recavano nel Vecchio Continente, vivevano l'emigrazione come un'esperienza temporalmente limitata al periodo necessario per concretare quel progetto di accumulazione monetaria che, nelle loro intenzioni, avrebbe dovuto migliorare, una volta tornati al paese d'origine, le proprie condizioni socio-economiche.

${ }^{5}$ Cf. Lonni, A., Mondi a parte. Gli immigrati tra noi, Paravia, Torino, 1999, p. 37.

${ }^{6}$ Cf. Melotti, U., «Migrazioni internazionali e integrazione sociale: il caso italiano e le esperienze europee», in Delle Donne, M., Melotti, U., Petilli, S. (a cura di), Immigrazione in Europa. Solidarietà e conflitto, CEDISS (Centro Europeo di Scienze Sociali), Roma, 1993, p. 37.

7 «Islam in Europa e in Italia», numero monografico della rivista XXI secolo - Studi e ricerche della Fondazione Giovanni Agnelli 2 (1994), p. 3. Nello stesso senso cf. anche Perocco, F., «L'Italia nella costruzione dell'islam europeo», in Saint-Blancat, C. (a cura di), L'islam in Italia. Una presenza plurale, Edizioni Lavoro, Roma, 1999, pp. 48-49.

${ }^{8}$ Cf. Ivi, p. 49. 
Un'immigrazione così intesa, cioè motivata da ragioni squisitamente economiche e vissuta nella congettura di un ritorno, faceva sì che le strategie di inserimento degli immigrati nella società di accoglienza fossero minime, limitate all'ambito lavorativo. Questo spiega perché i musulmani non avanzassero, nel periodo considerato, richieste volte alla pubblica esplicitazione della propria appartenenza all'islam, preferendo piuttosto relegare quest'ultimo alla dimensione privata e domestica. ${ }^{9}$

La crisi petrolifera del $1973^{10}$ svuotò la costruzione illusoria formatasi negli anni del dopoguerra europeo attorno alle dinamiche migratorie.

La recessione economica, conseguente alla carenza di «oro nero», provocò, nei paesi europei importatori di manovalanza, un notevole incremento del tasso di disoccupazione sia tra gli autoctoni che tra gli stranieri. Per far fronte a questa situazione furono adottate, da un lato, misure volte a chiudere le porte ad ogni ulteriore afflusso di lavoratori non comunitari, dall'altro, senza successo, politiche finalizzate a favorire il ritorno in patria degli immigrati.

Per questi ultimi, infatti, la chiusura delle frontiere nordeuropee e le politiche che miravano a favorire il rimpatrio nei paesi d'origine, hanno contribuito a modificare la percezione dell'esperienza migratoria: l'impossibilità di porre in essere percorsi migratori temporanei o cadenzati da ritorni periodici-in quanto se fossero tornati nei propri paesi non avrebbero avuto più la possibilità di rientrare in Europa-li spinse a prendere coscienza del fatto che, viste le scarse attrattive economiche degli Stati di provenienza, rimaneva loro un'unica opzione, ossia lo stanziamento definitivo nei paesi del Vecchio Continente dove avevano vissuto fino ad allora. ${ }^{11}$

Questa presa di coscienza ebbe come diretta conseguenza l'innescarsi del terzo dei quattro cicli migratori. Fino a quando l'emigrazione venne percepita come un'esperienza limitata nel tempo, a raggiungere l'Europa furono solo gli uomini, mentre mogli e figli restavano nei paesi d'origine. Nel momento in cui, invece, l'emigrazione mutava da temporanea

\footnotetext{
${ }^{9}$ Cf. Dassetto, F., «Il nuovo Islam europeo», in Ferrari, S. (a cura di), L'Islam in Europa. Lo statuto giuridico delle comunità musulmane, Il Mulino, Bologna, 1996, p. 20.

${ }^{10}$ Sulla crisi petrolifera del 1973, cf. Guarracino, S., Storia degli ultimi cinquant'anni. Sistema internazionale e sviluppo economico dal 1945 ad oggi, Bruno Mondadori, Milano, 1999, pp. 317-321.

${ }^{11}$ Cf. Lonni, A., Mondi a parte, p. 39; Bonifazi, C., L'immigrazione straniera in Italia, Il Mulino, Bologna, 1998, p. 69.
} 
in permanente, e quindi i paesi nordeuropei divenivano l'orizzonte di vita definitivo degli immigrati, costoro si adoperarono affinché venissero raggiunti dai propri familiari, possibilità questa garantita dall'istituto del ricongiungimento familiare. ${ }^{12}$ Ecco perché questo terzo ciclo migratorio fu caratterizzato prevalentemente dalla presenza di donne e bambini e si sviluppò con caratteristiche molto diverse da quelle precedenti: "se nelle due fasi precedenti i rapporti interessavano solo l'ambito strettamente economico e professionale, con la ricostruzione dei nuclei familiari, la presenza delle donne, la crescita delle nuove generazioni, sorgono problemi e bisogni nuovi, attinenti ai vari ambiti della vita, che diventano altrettante occasioni per avviare relazioni più ampie e diversificate con $\mathrm{i}$ diversi settori della società e con le istituzioni dei vari paesi". ${ }^{13}$ È negli anni '70, quindi, che prende il via quel graduale processo mediante il quale l'immigrato passa da un'identificazione etno-nazionale ad una prettamente religiosa (un processo che col passar del tempo perderà il suo carattere di gradualità, per acquisire quello di immediatezza. Questa avvenuta evoluzione trova nell'islam italiano il suo esempio concreto). Il mutamento dei progetti migratori degli immigrati musulmani maschiche ha come conseguenza la presenza in Europa delle loro famiglie e quindi anche di giovani da educare e da socializzare all'islam-e la scomparsa della funzione economica di tali migrazioni ${ }^{14}$ — che vanifica lo scopo dell'emigrazione stessa, ossia l'accumulazione monetaria ${ }^{15}$

\footnotetext{
12 "Esso veniva infatti garantito in tutti i paesi dell'Europa occidentale in virtù dell'adesione da parte di questi ad alcuni strumenti giuridici internazionali in materia di diritti umani quali, ad esempio, la Convenzione europea dei diritti dell'uomo che invoca il rispetto della vita familiare; la Dichiarazione universale dei diritti dell'uomo che afferma che la «famiglia è l'unità naturale e fondamentale della società e dello stato» (art. 16); il Patto internazionale sui diritti civili e politici; e l'Atto finale della Conferenza di Helsinki sulla sicurezza e sulla cooperazione in Europa, che stabilisce norme per il ricongiungimento familiare"; cf. Collinson, S., Le migrazioni internazionali, p. 121, nota 62. Circa il fatto che i flussi che costituivano la nuova fase del ciclo migratorio islamico fossero dovuti in prevalenza da ricongiungimenti familiari, cf. Melotti, U., «Migrazioni internazionali», p. 58.

13 Pacini, A., «I musulmani in Italia», pp. 23-24.

14 Perdita causata dall'alto tasso di disoccupazione conseguente alla recessione economica di quegli anni.

${ }^{15}$ Cf. Dassetto, F., Bastanier, A., Europa: nuova frontiera dell'Islam, pp. 117-118; Dassetto, F., L'Islam in Europa, Edizioni della Fondazione Agnelli, Torino, 1994, pp. 65-66; Allievi, S., Dassetto, F., Il ritorno dell'islam. I musul-
} 
ebbero, come corollario, il rafforzamento di quella domanda di islam che fino ad allora era stata molto debole. ${ }^{16}$ Vengono, quindi, avanzate le prime istanze finalizzate ad un più adeguato inserimento di questa nuova presenza nelle istituzioni dei paesi europei. Queste ultime non incontrano particolari problemi a soddisfare le richieste più semplici, come ad esempio quelle relative al regime dietetico dei nuovi arrivati. Più difficile da soddisfare, in quanto interferiscono con vigore nella vita delle popolazioni autoctone, sono, invece, le richieste connesse all'esercizio di quelle pratiche cultuali per espletare le quali si rivela funzionale l'occupazione di «spazi» pubblici: "l' 'aid al-kebīr, per esempio, che vede nei quartieri musulmani tra comportamenti furtivi condurre in casa il montone che sarà ucciso dal capofamiglia. Condotte che rinviano alle società agrarie $\mathrm{e}$ pastorali e che incrinano la vita quotidiana. Il puzzo delle viscere versate nelle spazzature, le fognature che si occludono, pelli di montone da conciare; i vicini non musulmani che si inquietano; le società protettrici di animali che gridano, qua o là, alla barbarie. Un modus vivendi dovrà essere trovato un po' alla volta. Ma non c'è ancora. Come per il periodo di ramadan, il nono mese dell'anno islamico, effervescente, col tempo quotidiano che si rovescia tra giorno e notte. Che, in particolare allorché avviene nel periodo estivo, è all'origine di notti calde e agitate, dove l'allegria degli uni si scontra con l'irritazione degli altri". ${ }^{17}$

Senza risposta rimane, poi, la richiesta di introdurre, mediante corsi di religione islamica o con l'apertura di scuole islamiche, l'islam nello spazio scolastico. ${ }^{18}$

A partire dagli anni ottanta si assiste alla nascita di un nuovo ciclo migratorio, il quarto. Questo, rispetto ai precedenti, appare poco definito sia

mani in Italia, Edizioni Lavoro, Roma, 1993, pp. 29-30.

${ }^{16}$ Cf. Ivi, p. 29; Dassetto, F., «Il nuovo Islam europeo», p. 20.

17 Allievi, S., Dassetto, F., Il ritorno dell'islam, p. 30.

18 "Sembra però che la preoccupazione di trasmettere l'islam, più forse come nucleo di valori che come fede, interessa l'insieme della popolazione musulmana perché il fallimento della socializzazione, soprattutto nella scuola, e i fenomeni di deviazione colpiscono pesantemente le giovani generazionisoprattutto maschili-e mettono ampiamente in discussione l'azione di socializzazione non musulmana, nelle quali le famiglie avevano riposto molte speranze. Emerge quindi in maniera crescente la convinzione della necessità di ritornare ad una socializzazione islamica, la sola che possa indicare la «retta via» ai giovani che si sono smarriti"; cf. Dassetto, F., L'Islam in Europa, p. 101. 
per il venir meno dei legami esclusivi tra i paesi di partenza e quelli di arrivo, ${ }^{19}$ sia per la presenza non solo di immigrati economici ma anche di rifugiati. ${ }^{20}$ Altro elemento di differenziazione è individuabile nel fatto che, a partire da questi anni, gli immigrati si dirigono, spesso clandestinamente, ${ }^{21}$ verso quei paesi (Italia, Spagna, Grecia e Portogallo) ritenuti non molto attraenti dal punto di vista economico, ${ }^{22}$ ma facilmente accessibili perché privi di legislazione riguardante l'immigrazione o perché i controlli all'ingresso sono facilmente eludibili. Tuttavia, proprio per queste caratteristiche, gli Stati della sponda meridionale dell'Europa vengono spesso considerati, dagli immigrati, come paesi di primo accesso, da cui possibilmente proseguire il loro viaggio in direzione delle più appetibili regioni del Nord Europa. Essendo però effettuato, da queste ultime, un severo controllo, di solito Spagna ed Italia rimangono luogo definitivo di immigrazione e vi si innescano le fasi successive del ciclo migratorio, in cui gli immigrati intrecciano relazioni molteplici con la società di accoglienza attuando la loro stabilizzazione al suo interno.

Pacini sottolinea come "il carattere complesso e fluido di questo ciclo si è ulteriormente rafforzato a partire dagli inizi degli anni novanta, con la formazione di nuovi consistenti flussi provenienti dai Balcani in seguito al crollo dei regimi comunisti. Si tratta anche in questo caso di flussi difficilmente controllabili, in cui i motivi economici, sociali e politici si sovrappongono, per la presenza di situazioni conflittuali nell'area, si pensi alla recente guerra del Kosovo, o per il collasso delle istituzioni dello Stato e della società, come è avvenuto in Albania. Situazioni similari spingono però all'emigrazione anche in altre aree, in particolare il Kurdistan turco ed iracheno. Una novità di questi flussi è costituita dalla composizione demografica: i nuclei familiari costituiscono una percentuale più importante al loro interno, per cui arrivano in Europa, e soprattutto in Italia, donne e bambini, accanto a uomini, come

\footnotetext{
${ }^{19}$ Secondo Perocco, "adesso dal Maghreb, dall'Africa sub-sahariana, dal Medio Oriente, l'emigrazione prende la direzione di tutti i paesi europei, anche di quelli in cui non ci sono antiche relazioni coloniali, affinità linguistiche, a volte reti comunitarie già operanti”; Perocco, F., «L'Italia nella costruzione dell'islam europeo», p. 50.

${ }^{20}$ Sulla distinzione tra richiedenti asilo e rifugiati, cf. Collinson, S., Le migrazioni internazionali, pp. 55-56.

${ }^{21}$ Cf. Ivi, pp. 125-126; Perocco, F., «L'Italia nella costruzione dell'islam europeo», p. 51.

${ }^{22}$ Cf. Melotti, U., «Migrazioni internazionali», p. 42.
} 
primi immigrati. Inoltre, dal punto di vista dell'appartenenza culturale, ancora una volta predomina la composizione islamica". ${ }^{23}$

A partire dagli anni ottanta, cambia il profilo dei nuovi immigrati: "se fino alla metà degli anni Settanta prevaleva quasi esclusivamente una popolazione rurale ed analfabeta, ora si accentua considerevolmente la parte di popolazione giovane, urbana ed alfabetizzata. ${ }^{24}$ Popolazioni create dallo sviluppo del sistema scolastico nei paesi di provenienza con deboli sbocchi professionali. Popolazione attratta sia da una «socializzazione anticipata» verso il consumo, sia da una socializzazione verso il lavoro. Sono queste popolazioni che hanno fatto sorgere e sviluppare l'islam europeo". 25

Negli anni ottanta e novanta l'appartenenza islamica comincia ad essere espressa in maniera sempre più esplicita dalle popolazioni immigrate musulmane, che proseguono così quel cammino di visualizzazione e di istituzionalizzazione avviato già negli anni settanta. La manifestazione della adesione al messaggio di Allah, da parte degli immigrati, avviene mediante una serie di richieste, soddisfacendo le quali si arriverebbe ad ottenere per l'islam uno statuto paragonabile a quello che gli Stati europei hanno concesso alle altre confessioni religiose, inserendolo così tra le componenti del sistema socio-giuridico europeo. Le richieste in questione possono essere così riassunte:

1. rivendicazioni che hanno delle ricadute sull'organizzazione e la gestione degli spazi pubblici urbani: costruzione di moschee, creazione di spazi cimiteriali destinati alle sepolture rituali islamiche, concessione di spazi idonei per la macellazione rituale;

2. rivendicazioni concernenti l'organizzazione del lavoro consistenti nella richiesta di orari e calendari flessibili che permettano ai credenti di onorare gli obblighi religiosi (le cinque preghiere quotidiane, ramaḍ̄an, festività religiose);

3. rivendicazioni riguardanti l'organizzazione del sistema educativo:

${ }^{23}$ Pacini, A., «I musulmani in Italia», pp. 24-25.

${ }^{24}$ Nello stesso senso, cf. Perocco: "si verificano mutamenti strutturali nelle società di partenza che condizionano totalmente le pratiche ed i progetti migratori dei nuovi immigrati musulmani. La nuova immigrazione è infatti composta da popolazione prevalentemente giovane, urbana e alfabetizzata, al contrario dei flussi del passato, composti per la maggior parte da una popolazione rurale e analfabeta"; Perocco, F., «L'Italia nella costruzione dell'islam europeo», p. 50.

${ }^{25}$ Cf. Dassetto, F., Bastanier, A., Europa: nuova frontiera dell'Islam, pp. 253-254. 
l'insegnamento della religione islamica nella scuola pubblica, la possibilità di aprire scuole private islamiche, il ritorno ad un'organizzazione scolastica separata in base al sesso, la possibilità di indossare segni di appartenenza culturali nelle classi (per esempio il hị̆āb);

4. rivendicazioni finalizzate ad ottenere l'applicazione dello statuto personale islamico alle controversie familiari che coinvolgono $i$ musulmani a prescindere dalla loro cittadinanza. ${ }^{26}$

Per quanto riguarda le problematiche gestibili a livello locale, ossia quelle elencate al punto a, le risposte delle istituzioni europee sono state positive; in merito alle altre richieste, invece, il cui espletamento ricade nella competenza delle amministrazioni locali—sia pur nel quadro delle leggi nazionali-o degli organi centrali dello Stato, sorgono problemi, oltre che di ordine specificamente giuridico, anche di ordine pratico: si pensi alle notevoli difficoltà incontrate dagli Stati europei di immigrazione nell'individuare, anche tra i musulmani in essi residenti, così come è avvenuto in precedenza per gli appartenenti alle altre confessioni reli-

${ }^{26}$ Quest'ultima richiesta, nella sua formulazione esplicita, è stata avanzata solo in Gran Bretagna. "Il primo tentativo volto ad introdurre nell'ordinamento giuridico inglese uno statuto personale islamico è stato compiuto alla conferenza organizzata a Birmingham nel 1975 dall'Union of Muslim Organizations (UMO) del Regno Unito e dell'Eire; da allora questa proposta è stata ribadita in numerose altre occasioni. Recentemente, una simile richiesta è stata presentata nel «Muslim Manifesto» pubblicato dal Muslim Institute. Contemporaneamente alla domanda dell'UMO di introdurre un sistema di statuto personale, Zaki Badawi ha predisposto un modello di testamento diretto a consentire ai musulmani di disporre delle loro proprietà in modo conforme sia alla legge islamica che a quella inglese: ma il progetto di Badawi non ha suscitato alcun interesse. Fino ad oggi nessun governo britannico ha mostrato la minima propensione ad introdurre uno statuto giuridico personale per i musulmani”; cf. Bradney, A., «Lo statuto giuridico dell'Islam nel Regno Unito», in Ferrari, S. (a cura di), L'Islam in Europa, pp. 182-183. Cf. anche Nielsen, S. J., «Il diritto familiare nelle rivendicazioni delle popolazioni musulmane in Europa», in AA. VV., I musulmani nella società europea, Edizioni della Fondazione Agnelli, Torino, 1994, pp. 79-89. Sulla complessa problematica relativa alla stipula di un'intesa tra lo Stato italiano e la confessione islamica, si veda il recente libro di Cilardo, A., Il diritto islamico e il sistema giuridico italiano. Le bozze di intesa tra la Repubblica Italiana e le Associazioni islamiche italiane, Presentazione di Borrmans, M., Introduzione di Musselli, L., Edizioni Scientifiche Italiane, Napoli, 2002. 
giose, interlocutori rappresentativi di tutta la comunità islamica locale con cui trattare le questioni connesse al suo status giuridico. Le difficoltà riscontrate da parte degli Stati europei nell'individuare tali interlocutori sono strettamente connesse al carattere di frammentarietà che connota le organizzazioni islamiche europee. "Questa difficoltà è dovuta ad un fatto strutturale all'interno dell' 'islam, che non conosce, ad eccezione dell' islam sciita, alcuna forma di clero o di gerarchia connessa al culto. Poiché tradizionalmente nell'islam la sfera prettamente religiosa e la sfera temporale sono strettamente unite e si legittimano reciprocamente, in concreto è l'autorità politica, religiosamente legittimata, a controllare e gestire tutto l'apparato religioso. Negli Stati musulmani moderni questo avviene tramite il ministero per gli Affari religiosi. Nell'ambito dell'emigrazione musulmana in Europa le situazioni tuttavia diventano assai complesse: da un lato nelle società europee la sfera dello Stato e quella delle confessioni religiose sono distinte ed indipendenti, per cui lo Stato non gestisce organismi o attività religiose. Dall'altra i musulmani presenti in Europa provengono da una molteplicità di Stati e appartengono a una varietà di movimenti e correnti diverse, per cui diventa arduo per loro esprimere una rappresentanza unitaria". ${ }^{27}$

\section{L'ESPERIENZA ITALIANA}

L'immigrazione islamica in Italia presenta, rispetto a quella d'Oltralpe, più differenze che analogie.

Diversi sono stati, innanzitutto, i tempi e le modalità del rispettivo divenire.

Infatti, differentemente dai paesi nordeuropei, "l'Italia è diventata terra di immigrazione senza volerlo e senza neppure saperlo: una decisione che si sono incaricati di prendere i fatti anziché la politica. Alla metà degli anni ' 70 , a partire dalla chiusura delle frontiere decise dalle nazioni di antica, tradizionale immigrazione del Centro-Nord europeo, in risposta alla crisi provocata dal primo shock petrolifero, il nostro paese divenne per un disorientato esercito di immigranti quello che in gergo gli esperti chiamano un second-best, un'alternativa meno gradita ma praticabile". ${ }^{28}$ L'immigrazione straniera in Italia è iniziata, quindi, in un periodo, la seconda metà degli anni settanta, in cui la domanda di lavoro si riduceva drasticamente e aumentava per contro, e in misura consi-

\footnotetext{
${ }^{27}$ Cf. Pacini, A., «I musulmani in Italia», p. 29.

${ }^{28}$ Cf. Bolaffi, G., Una politica per gli immigrati, Il Mulino, Bologna, 1996, p. 31 .
} 
stente, la disoccupazione. Si spiega così la singolare caratteristica del fenomeno immigratorio italiano rispetto a quello in precedenza registrato nei paesi nordeuropei. In questi ultimi, infatti, l'ingresso della forza lavoro straniera fu, dallo Stato, guidata e concentrata nelle aree di elevata industrializzazione dove, a causa della piena occupazione, le fabbriche non trovavano più braccia disponibili tra i lavoratori nazionali. Viceversa, in Italia gli immigrati non solo sono arrivati «spontaneamente», offrendosi ad un sistema produttivo che non si era ancora reso conto di averne bisogno, ma hanno trovato lavori ed occupazione, in gran parte precari ed illegali, prima nell'area meridionale della penisola, meno industrializzata e a più forte tensione occupazionale, e poi nei grandi centri urbani. ${ }^{29}$ Se ne deduce, di conseguenza, che l'immigrazione straniera verso lo «Stivale» ha mosso i primi passi quando oramai appariva superata la fase dei flussi ordinati e univocamente direzionati, che nascevano dal reciproco e a volte esplicito (in termini di legislazione, di servizi pubblici apprestati) interesse economico del paese di emigrazione e di quello di immigrazione. Si può dunque dire, riassumendo, che il movimento migratorio verso l'Italia, così come verso gli altri paesi dell'Europa meridionale, sia stato caratterizzato dal fatto di sorgere in concomitanza con il declino del ruolo trainante della grande industriache riduce dappertutto il suo contributo all'occupazione ${ }^{30}$ — quale fattore di attivazione della domanda di lavoro ${ }^{31}$ e del suo effetto di richiamo.

Nell'analizzare, quindi, i fattori che hanno portato la penisola italiana a diventare area di destinazione delle migrazioni extracomunitarie, piuttosto che ai fattori di attrazione (pull factor), un grande rilievo va assegnato alle cosiddette motivazioni esterne, riconducibili, oltre che alle restrittive politiche migratorie adottate dai tradizionali paesi europei di accoglimento, anche all'enorme effetto spinta attribuito ai fattori di espulsione (push factor) nei paesi di partenza. ${ }^{32}$

${ }^{29}$ Cf. Ivi, pp. 31-32; Pugliese, E., «L'immigrazione», in AA. VV., Storia dell'Italia repubblicana, vol. III, tomo I, Einaudi, Torino, 1996, pp. 934-935; Tonizzi, M. E., Le correnti migratorie del '900, Paravia, Torino, 1999, p. 143.

${ }^{30} \mathrm{Da}$ questo punto di vista, Pugliese fa notare come l'Italia stia invece cominciando a rappresentare oggi un caso particolare, dato il crescente inserimento degli immigrati nelle piccole aziende; cf. Pugliese, E. (a cura di), Rapporto immigrazione. Lavoro, sindacato, società, Ediesse, Roma, 2000, p. 15.

${ }^{31} \mathrm{Cf}$. Ibidem.

${ }^{32}$ Cf. Harrison, G., «Antropologia culturale dei processi migratori e i diritti umani», in Damiano, E. (a cura di), Homo migrans, Franco Angeli, Milano, 
Analogamente a quanto già avvenuto negli altri paesi europei, anche in Italia i primi ad arrivare sono gruppi composti, quasi esclusivamente, da maschi. Si tratta di nuclei di immigrati tunisini che trovano occupazione in Sicilia nel settore della pesca, in particolare presso il porto di Mazara del Vallo, e, in misura inferiore, in quello agricolo, dove vengono impiegati soprattutto nel Ragusano, località in cui sono molto diffuse le coltivazioni in serra. ${ }^{33}$ Successivamente si comincia a segnalare l'arrivo dei marocchini, i quali si distribuiscono su tutto il territorio nazionale, dedicandosi, soprattutto nelle regioni meridionali, al commercio ambulante. Gli egiziani, ${ }^{34}$ invece, che in questa fase sono il gruppo nazionale maggioritario, si impiegano nell'area della piccola impresa del nord. ${ }^{35}$

Un altro elemento che contribuisce a rendere peculiare il caso italiano nel panorama dell'islam europeo è una presenza etno-nazionale forte-

1998, pp. 132-136; Melchionda, E., «Il paradosso italiano», in Delle Donne, M., Melotti, U., Petilli, S., Immigrazione in Europa, pp. 185-186; Sgroi, E., "L'impatto economico della immigrazione: teoremi, metafore, realtà», in Sociologia urbana e rurale 60 (1999), p. 35; Natale, M., Strozza, S., Gli immigrati stranieri in Italia, Cacucci, Bari, 1997, pp. 213-214. Diversamente per Bonifazi, "l'aumento dei fattori espulsivi spiega solo parzialmente questa dinamica del fenomeno che trova, infatti, all'altro polo, una permanenza, sia pur su parametri qualitativi e quantitativi differenziati, di fattori di richiamo, o almeno di potenziale assorbimento, di natura economica. Settoriali e circoscritti, non più basati su uno squilibrio quantitativo complessivo dei sistemi economici, ma sulla permanenza, in un quadro di generale eccesso di offerta, di squilibri settoriali, a volte di grande intensità, legati ai processi di segmentazione territoriale e settoriale del mercato del lavoro. In questo senso la situazione italiana rappresenta un esempio assai significativo, anche perché la segmentazione del mercato del lavoro e gli squilibri economici settoriali assumono un'articolazione territoriale che trova, con ogni probabilità, pochi riscontri all'estero, non solo nella ben nota direttrice Sud-Nord, ma anche per ambiti geografici molto più ristretti e circoscritti, dato che, specie nell'Italia centrosettentrionale, la differenziazione assume una veste microterritoriale, con forti connotati di specializzazione produttiva tra aree anche contigue"; Bonifazi, C., L'immigrazione straniera in Italia, pp. 175-176.

33 Sull'immigrazione tunisina in Sicilia, cf. Slama, H., ...e la Sicilia scoprì l'immigrazione tunisina, INCA-CGIL Sicilia, Palermo, 1986.

${ }^{34}$ Sulle politiche migratorie egiziane, cf. Mancini, L., Immigrazione musulmana e cultura giuridica. Osservazioni empiriche su due comunità di egiziani, Giuffrè, Milano, 1998, pp. 80-93.

35 Cf. Pugliese, E., «L’immigrazione», pp. 937-938. 
mente variegata. Infatti, sebbene, oggi, la comunità con il più alto tasso di crescita sia rappresentata dai marocchini, la popolazione immigrata islamica residente in Italia è caratterizzata dalla presenza di altre consistenti componenti etniche e nazionali, quali ad esempio quella albanese e senegalese. Tra i musulmani immigrati nella penisola italiana manca, perciò, a differenza di quanto avviene in paesi come Francia, Germania e Gran Bretagna dove esiste una matrice etnica dominante nell'immigrazione-maghrebini per la Francia, indopakistani per la Gran Bretagna e turchi per la Germania-un gruppo etno-nazionale in grado di monopolizzare il campo immigratorio. ${ }^{36} \mathrm{La}$ frammentazione etnica è dovuta nel caso italiano a due fattori. "L'Italia non ha mai trattenuto relazioni privilegiate con le sue ex colonie: ${ }^{37}$ non si è mai verificata un'immigrazione massiccia dalla Somalia, dall'Etiopia o dalla Libia. Il sistema coloniale italiano era basato su un assetto di protettorato o di indirect rule, fatto che ha relativamente ammorbidito tutta la fase della decolonizzazione. Quello stesso processo, nel subcontinente indiano o in Algeria, ha provocato gravi crisi politiche-la spaccatura fra India e Pakistan nel 1947 alla vigilia dell'indipendenza, la guerra di liberazione coloniale fra il 1954 e 1962 tra Francia e Algeria-che hanno messo in moto già all'epoca importanti flussi migratori verso l'Europa. L'altro fattore risiede nella particolare collocazione geopolitica dell'Italia, con il suo doppio versante mediterraneo, quello balcanico e quello arabo-africano, che comprime il paese fra due spinte, una da sud l'altra da est investendo quest'ultima sia il litorale adriatico che la terraferma, in particolare la zona di Gorizia. Confinando con la Slovenia, l'Italia funge da ponte per l'immigrazione vicino-mediorientale e balcanica, dal momento che la Bosnia non richiede agli stranieri il visto d'ingresso". 38

Va rilevato che la diversità delle provenienze geografiche non è scevra di conseguenze. Infatti, l'immigrato musulmano che risiede in Italia, così come in ogni altro paese non islamico, vive, a seconda del paese da cui proviene, un diverso rapporto tra ordinamento statale e ordinamento reli-

\footnotetext{
${ }^{36}$ Cf. Pace, E., Perocco, F., «L'Islam plurale degli immigrati in Italia», in Studi emigrazione, anno XXXVII, marzo, 137 (2000), p. 4.

${ }^{37}$ Cf. Giannasi, A., «Musulmani in Italia», in Africa 2 (2000), <http://www. cadr.it/islam/00-2-giannasi.htm> (02 aprile 2003).

${ }^{38}$ Cf. Fouad Allam, K., «L'Islam contemporaneo in Europa e in Italia fra affermazione identitaria e nuova religione minoritaria», in Zincone, G. (a cura di), Secondo rapporto sull'integrazione degli immigrati in Italia, Il Mulino, Bologna, 2001, pp. 334-335.
} 
gioso. Potrebbe infatti arrivare da uno di quei paesi (Arabia Saudita, Iran, Pakistan, Sudan, Afghanistan) in cui esiste una coincidenza pressoché perfetta tra legge religiosa e legge statale; oppure da Stati (Egitto, Marocco, Siria, Iraq) in cui la legge religiosa, pur costituendo in misura più o meno rilevante uno dei fondamenti della legge civile, viene però da quest'ultima recepita solo parzialmente; o, infine, giungere da Tunisia, Turchia, Senegal, dove la legge statale è completamente, o comunque in larga parte, indipendente da quella religiosa.

Nonostante la ridotta consistenza numerica e il carattere recente dell'insediamento, le comunità islamiche italiane, diversamente da quelle nordeuropee, hanno manifestato sin dall'inizio una vitalità associativa e una «visibilità» sociale comparativamente piuttosto elevate: differentemente dai paesi d'Oltralpe connotati da una presenza islamica più matura, "le moschee, in Italia, sono moschee di prima generazione, volute e promosse da immigrati per lo più arrivati anche da relativamente poco tempo. In altri paesi europei la loro diffusione sul territorio è invece da mettere in relazione e in coincidenza con la presenza di una seconda generazione nei confronti della quale si sentiva l'urgenza di una trasmissione anche religiosa". ${ }^{39}$ Il ridursi dei tempi sociali connessi alla visibilità ${ }^{40}$ è attribuibile in particolare, secondo Guolo, all'emergere di una leadership comunitaria, di marcato orientamento «islamico», che rivendica la piena visibilità sociale dell'islam nella scena pubblica e rifiuta di ridurlo alla sfera privata. ${ }^{41} \mathrm{Si}$ tratta di immigrati che, infatti, a differenza

${ }^{39}$ Cf. Allievi, S., «Complessità e dinamiche dell'islam in Italia», in El Ayoubi, M., Islam plurale, Edizioni com nuovi tempi, Roma, 2000, p. 95.

40 "Se era intuibile che prima o poi l'Islam si sarebbe manifestato con forza in seno alle comunità immigrate anche nel nostro paese (per capirlo bastava dare un'occhiata anche distratta a quanto stava accadendo oltre confine dal lato nord, negli altri paesi europei), non altrettanto si può dire della velocità con cui questo è accaduto: superiore, senza dubbio, a quella osservata in altre realtà europee. L'islamizzazione dell'immigrazione, per usare una terminologia anche troppo forte, è avvenuta in Italia più in fretta che altrove: quando gli immigrati si erano appena stabiliti, quando avevano appena disfatto le valigie. L'Islam è diventato subito, o quasi, una componente importante del processo di socializzazione degli immigrati; e molto presto una componente visibile anche all'esterno, nello spazio pubblico"; Allievi, S., «Dall'Islam ai musulmani. Fare ricerca su una 'religione immigrata'», in Sociologia urbana e rurale 58 (1999), p. 112. Cf. anche Pace, E., Perocco, F., «L'Islam plurale», p. 5.

${ }^{41}$ Cf. Guolo, R., «Attori sociali e processi di rappresentanza nell'islam italiano», in Saint-Blancat, C. (a cura di), L'islam in Italia, p. 67; Fouad Allam, K., 
dei loro predecessori, sono per la maggior parte giovani, di origine urbana, alfabetizzati e, cosa più importante, cresciuti in quei paesi nordafricani e del Vicino Oriente (Algeria, Siria, Egitto) che negli ultimi trent'anni hanno conosciuto il cosiddetto «risveglio islamico», ${ }^{42}$ caratterizzato dal fenomeno del «ritorno alla moschea» e dalla richiesta di islamizzazione della società. ${ }^{43}$ Ecco perché tali attori-oltre a soddisfare, riproponendone la ritualità, i bisogni della fede dei propri correligionari-sono capaci di negoziare con l'ambiente circostante quelle strategie tese a ricreare una comunità in senso islamico. ${ }^{44}$ Infatti "il loro primo obiettivo è quello di definire un contesto spaziale e simbolico in cui il musulmano possa, attraverso il contatto e l'immersione nella purezza della comunità del Profeta, la umma ${ }^{45}$ ridurre il senso d'isolamento e riadattare i propri codici simbolici necessari per affrontare, saldamente orientati alla religione, l'esperienza dell'immigrazione in Occidente". 46 "La costruzione sociale dell'individuo necessita [quindi], anche simbolicamente, della separatezza (hiğra). Così le leadership islamiste [sic! $]^{47}$ incoraggiano la visibilizzazione di stili di vita e di un sistema dei segni contrapposti ai modelli della cultura dominante circostante. Il velo per le donne, la barba e la stessa ğalläbiyya (la lunga veste bianca) indossata dagli uomini, ogni qualvolta è possibile e comunque nel tempo della preghiera e delle ritualità canoniche, sono tutti elementi che mirano a rinforzare il «noi» sotteso all'identità islamica". ${ }^{48}$ Insomma, sono leader

«L'islam contemporaneo», p. 328.

${ }^{42}$ Cf. Allievi, S., Dassetto, F., Il ritorno dell'islam, pp. 132-134.

${ }^{43}$ Cf. Guolo, R., «È possibile un partito islamico in Italia?», in Limes 4 (1997), pp. 271-272; Giannasi, A., «Musulmani in Italia»; Fouad Allam, K., «L'islam contemporaneo», pp. 324-326.

${ }^{44}$ Cf. Guolo, R., «Attori sociali», p. 69.

${ }^{45}$ Sulla concezione della umma nell'islam dell'immigrazione, cf. Fouad Allam, K., «L'islam contemporaneo», pp. 326-328. Sulle implicazioni giuridiche del concetto di umma, si veda Cilardo, A., «La comunità islamica», in Ende, W., Steinbach, U. (a cura di), L'islam oggi, Edizioni Dehoniane, Bologna, 1993, pp. 13-42.

${ }^{46}$ Cf. Guolo, R., «Attori sociali», p. 71.

${ }^{47}$ Guolo traduce il termine francese islamiste, che in quella lingua indica $\mathrm{i}$ movimenti islamici radicali, con l'italiano islamista; tale termine, però, nella nostra lingua vuol dire «studioso di cultura islamica».

${ }^{48} \mathrm{Cf}$. Guolo, R., «Attori sociali», p. 74; Id., «Europa, terra d'Islam», in $I l$ Mulino, anno XLVIII, maggio - giugno, 1999, p. 548; nonché Melfa, D., 
la cui preoccupazione principale è quella di trasmettere un sistema di valori e norme di comportamento relativi alla condotta del musulmano nel nuovo ambiente intriso di «tentazioni»: ateismo e materialismo, ineguaglianza sociale, diffusione massiccia della criminalità, depravazione sessuale, droga e alcolismo, prostituzione; essi vi contrappongono «la civiltà dell'islam». ${ }^{49} \mathrm{~A}$ perseguire una strategia di inserimento di tipo comunitario sono, dunque, prevalentemente i movimenti «islamici», ${ }^{50}$ tra cui un ruolo di spicco è svolto dai Fratelli Musulmani (al-iḩwān almuslimūn), ${ }^{51}$ alla cui ideologia si ispirano i dirigenti di quelle moschee che sono simpatizzanti o aderenti all'Unione delle Comunità Islamiche in Italia (UCOII $\left.{ }^{52}\right) .53$

I movimenti «islamici» non esauriscono, però, la tipologia delle forme attraverso cui gli immigrati musulmani esprimono la loro appartenenza

«L'Islàm a Catania», in La critica sociologica, luglio - settembre, 130 (1999), pp. 63-64.

${ }^{49} \mathrm{Cf}$. Guolo, R., «Attori sociali», p. 70; Id., «È possibile un partito islamico in Italia», p. 278; Id., «Europa, terra d'Islam», p. 548.

${ }^{50}$ Di diverso avviso è invece Allievi per il quale "per un paradosso solo apparente, questi movimenti politicamente più radicali svolgono in realtà un ruolo implicitamnete «conservatore» e «calmieratore», più di freno che di acceleratore, sulle tematiche legate all'immigrazione e ai diritti dei musulmani, in parte perché timorosi di reazioni, ma soprattutto perché il loro centro di interessi si è «esternalizzato, legato com'è alla situazione dei paesi di origine piuttosto che di quello di accoglienza"; cf. Allievi, S., «Complessità e dinamiche», p. 98.

${ }^{51}$ Sui Fratelli Musulmani, cf. Cilardo, A., «Su alcune recenti formazioni islamiche», in Goldziher, I., Lezioni sull'islām, Edizioni Scientifiche Italiane, Napoli, 2000, pp. 325-333.

52 “L'Ucoii nasce nel 1990, per impulso di alcuni membri del Centro islamico di Milano [a sua volta nato, negli anni settanta, per iniziativa dell'Unione studenti musulmani in Italia (Usmi) allora molto numerosi in Italia, e fondatore della prima moschea (anche in senso architettonico) italiana: quella di al-Rậmān o del Misericordioso, inaugurata nel 1988]. L'Unione, formata dall'associazione dei maggiori centri islamici italiani, è senza dubbio la struttura musulmana più diffusa nel territorio. Al suo radicamento ha contribuito anche l'aver «ereditato» le strutture nazionali [moschee, sale di preghiera] dell'Usmi [che ha visto, infatti, ridurre sempre più la propria influenza a causa del ridursi del numero di studenti musulmani che si recano in Italia]"; Guolo, R., «La rappresentanza dell'islam italiano e la questione delle intese», in Ferrari, S. (a cura di), Musulmani in Italia, pp. 70-71.

${ }^{53}$ Sulle moschee italiane vicine ai Fratelli Musulmani, cf. Pacini, A., «I musulmani in Italia», p. 44. 
all'islam. Quest'ultima, infatti, trova espressione in altre due forme organizzative: l'«islam degli Stati» e le confraternite religiose.

"L'azione degli Stati islamici consiste nell'insieme di quelle iniziative poste in essere dagli Stati allo scopo di promuovere la «versione» dell'islam da essi sostenuta e sulla quale molto spesso basano la propria legittimazione politica. L'esempio più evidente di questa azione è la grande moschea di Roma che è direttamente connessa con la diplomazia e con il governo di vari Stati islamici". ${ }^{54}$ Infatti, i costi per la costruzione della moschea di Monte Antenne, ${ }^{55}$ sede del Centro Culturale Islamico d'Italia, l'unico ad aver ottenuto, nel dicembre del 1974, il riconoscimento ufficiale da parte dello Stato, e ad avere nel proprio consiglio di amministrazione gli ambasciatori di molti Stati islamici sunniti presso l'Italia o presso la Santa Sede, ${ }^{56}$ sono stati prevalentemente sostenuti dall'Arabia Saudita tramite la Lega del mondo musulmano, "organizzazione saudita che ha la triplice finalità di assicurare il sostegno all'islam sul piano internazionale, soprattutto dove i musulmani rappresentano una minoranza, di promuovere la missione islamica presso i non musulmani in Europa e altrove, e di controllare il «tipo» d'islam praticato, influenzandolo per quanto possibile in senso conservatore". ${ }^{57}$ Particolarmente funzionale al perseguimento di quest'ultimo obiettivo è l'interpretazione dell'islam propria della dottrina wahhābita, ${ }^{58}$ che, basata sul significato letterale del Corano e sulla rigida applicazione della $\check{s} a r \bar{c} c$, propugna una versione conservatrice dell'islam per la quale la dimensione giuridica, politica e sociale del vivere devono essere religiosamente legittimate. Esempio concreto dell'applicazione della dottrina wahhābita è

\footnotetext{
${ }^{54}$ Cf. Ivi., p. 37.

${ }^{55}$ Cf. Allievi, S., «L'ombra di San Pietro», in Il Manifesto, 8 agosto 2000.

${ }^{56}$ Nella lotta per l'acquisizione della leadership dell' islam italiano, tale argomento è stato utilizzato dal Centro Islamico di Milano e della Lombardia per sostenere che il Centro Islamico Culturale d'Italia non sarebbe rappresentativo perché promosso dalle ambasciate, e siccome le ambasciate sono extraterritoriali, appartengono ai paesi d'origine, per cui si tratta di stranieri, che come tali non possono pretendere di rappresentare l'islam italiano. Cf. Allievi, S., «Organizzazione e potere nel mondo musulmano: il caso della comunità islamica di Milano», in AA. VV., I musulmani nella società europea, pp. 172173.

${ }^{57}$ Cf. Pacini, A., «I musulmani in Italia», p. 37.

${ }^{58}$ Sul movimento wahhābita e la sua dottrina, cf. Cilardo, A., «Su alcune recenti formazioni islamiche», pp. 307-309.
} 
appunto l'Arabia Saudita ${ }^{59}$ che, per questa ragione, sembra essere contestata, all'interno del Centro Culturale Islamico d'Italia, da altri Stati come l'Egitto, il Marocco e la Tunisia, che, perseguendo sul piano interno un indirizzo politico volto a realizzare una progressiva democratizzazione, cercano invece di tenere a freno ogni sorta di radicalismo, a tal fine promuovendo, anche tra i loro immigrati in Italia, un islam non militante e non troppo conservatore. Emblematico è, da questo punto di vista, il caso dei tunisini. "La Tunisia esercita un controllo sui propri immigrati soprattutto in Sicilia: celebre è il caso di Mazara del Vallo, in cui risiede una popolosa colonia tunisina, dotata dal governo di origine di una scuola elementare che segue i programmi ufficiali della madrepatria, con insegnamento in arabo e francese. A Mazara tuttavia l'ambiente tunisino è all'apparenza laicizzato e non esiste alcuna moschea. La mancanza di luoghi di culto islamici è dovuta probabilmente al fatto che i residenti tunisini continuano ad avere rapporti con il paese d'origine, in cui si recano per le principali festività religiose, e d'altra parte lo Stato tunisino sembra non incoraggiare l'apertura di moschee per timore che diventino luogo di «fondamentalismo». Laddove se ne richiede l'apertura esso si adopera per esercitare un controllo sulla loro gestione. Così in Sicilia lo Stato tunisino ha assunto la gestione della moschea di Palermo tramite un accordo con il governo regionale siciliano: l'ambasciata tunisina in Italia ne nomina l'imām e ne segue l'attività attraverso l'Associazione culturale islamica di Palermo". ${ }^{60}$ Analoghe sono le strategie adottate, sia pur in maniera più temperata, da altri Stati, come ad esempio il Marocco e l'Egitto.

Proponendo una strategia non imperniata sul radicalismo, l'azione di questi Stati, eccezion fatta per quella saudita, finisce con l'entrare in contrasto con la leadership islamica, sostenitrice, invece, di un islam militante. Il comunitarismo «islamico» conduce una lotta aperta anche nei confronti del cosiddetto «islam nascosto», ossia la «non visibilizzazione» religiosa scelta dalle confraternite (tarìqa, pl. țuruq) șüfì, che costituiscono la terza forma con cui si presenta l'islam in Italia. ${ }^{61} \mathrm{La}$

${ }^{59}$ Cf. Reissner, J., «Libia e Arabia Saudita», in Ende, W., Steinbach, U. (a cura di), L'islam oggi, Edizioni Dehoniane, Bologna, 1993, pp. 501-511.

${ }^{60}$ Cf. Pacini, A., «I musulmani in Italia», p. 39; Schmidt di Friedberg, O., Borrmans, M., «Musulmans et chrétiens en Italie», in Islamochristiana 19 (1993), pp. 160-161.

${ }^{61}$ Sulle confraternite, cf. Aluffi Beck-Peccoz, R., «L'islam delle confraternite», in Ferrari, S. (a cura di), Musulmani in Italia, pp. 59-62, nonché Speziale, 
tarīqa più importante è quella muride del Senegal cui appartengono la maggior parte (ca. il 70\%) dei senegalesi immigrati nel nostro paese. Questi ultimi "compaiono [sulla scena pubblica] esclusivamente in quanto «senegalesi», mettendo l'accento sulla dimensione etnonazionale, più che come musulmani. Lo si evince anche dal tipo di attività che essi svolgono, che ha finalità dirette più all'integrazione pluralistica e alla trasformazione dei concetti di cittadinanza nella società ospite, che alla rivendicazione religiosa comunitaria. Essi propongono dunque una rappresentazione identitaria legata alla cittadinanza piuttosto che all'identità religiosa". 62

Alle confraternite tradizionali bisogna poi aggiungere alcuni gruppi di ispirazione șüfi costituiti prevalentemente da convertiti italiani. L'esempio più noto di questi gruppi è la COREIS (Comunità Religiosa Islamica), già Associazione per l'informazione sull'islam in Italia, guidata dallo šayh Pallavicini, con sede a Milano.

Pacini fa notare come "i principali centri di competizione in Italia appartengano a ognuna delle tre grandi categorie in cui trova espressione l'islam organizzato: l'islam degli Stati è infatti rappresentato dal Centro Culturale Islamico d'Italia; l'islam militante è variamente rappresentato dalle moschee e dai centri islamici che aderiscono all'UCOII; l'ambito dell'islam süfì e l'area delle confraternite sono rappresentati dalla COREIS. A questi va poi aggiunta l'Associazione dei Musulmani Italiani (AMI), che ha come proprio carattere specifico quello di accettare come membri effettivi solo cittadini italiani e chi si proclama seguace dell'islam sunnita, in aperta polemica con i Fratelli Musulmani e con l'islam militante dell'UCOII". ${ }^{63}$ Anche in Italia, quindi, come nel resto

F., «I sentieri di Allah: aspetti della diffusione dell'Islam delle confraternite in Italia», in La critica sociologica, ottobre - dicembre, 135 (2000), pp. 10-32.

${ }^{62}$ Cf. Guolo, R., «Le tensioni latenti nell'islam italiano», in Saint-Blancat, C. (a cura di), L'islam in Italia, p. 167. Nella stessa pagina l'autore mette in evidenza come "su questa scarsa propensione alla visibilizzazione religiosa, incide probabilmente anche una sorta di interiorizzazione del concetto di separazione tra religione e politica, caratteristica storica di uno Stato come il Senegal, fortemente influenzato dal modello francese di laicità. Pur essendo religione maggioritaria, l'islam non è in Senegal religione di Stato. Piuttosto che dal comunitarismo islamista, la presenza dei senegalesi sulla scena pubblica italiana è caratterizzata dal «senegalismo» e da un panafricanismo improntato ai «diritti universali dell'uomo» che non si pone in contrasto con la cultura del paese ospitante".

${ }^{63}$ Cf. Pacini, A., «I musulmani in Italia», p. 48. 
d'Europa, si è di fronte ad una frammentazione e ad una competizione delle organizzazioni islamiche che rende difficile, per lo Stato italiano, il compito di trovare un interlocutore realmente rappresentativo con cui poter trattare le condizioni che stabiliscano lo status dei musulmani in Italia. L'impossibilità di produrre una rappresentanza unitaria dell'islam italiano è palesata dalla presentazione, da parte delle organizzazioni islamiche (ad eccezione del Centro Culturale Islamico d'Italia) di ben tre bozze d'intesa ${ }^{64}$ con cui si chiede di dare cittadinanza, all'interno dell'ordinamento giuridico italiano, ai tratti maggiormente espressivi dell'esperienza religiosa islamica. Le rivendicazioni contenute in tali bozze, che non sono molto diverse da quelle avanzate dai musulmani negli altri paesi europei, sono state, di recente, oggetto di attenta analisi da parte di un gruppo di studiosi di diritto ecclesiastico. ${ }^{65}$

Da questa analisi è emersa, innanzitutto, un'area in cui le richieste avanzate nelle bozze d'intesa possono essere accolte fin d'ora sulla base della legislazione vigente: in riferimento alla costruzione e manutenzione degli edifici di culto e alla concessione di reparti separati all'interno dei cimiteri nonché al rispetto delle esigenze religiose islamiche in materia di macellazione; per l'abbigliamento; con qualche limite, in ambito lavorativo; per buona parte della problematica scolastica e dell'assistenza spirituale nelle carceri, disciplinata dal recente regolamento di esecuzione della legge 26 luglio 1975, n. 354.

In altri casi è necessario un intervento legislativo, attuabile però nell'ambito della legislazione unilaterale dello Stato. In relazione al rispetto delle festività religiose e dei «tempi di preghiera», si potrebbe provvedere con una norma che rinvii ad accordi tra lavoratori e datori di lavoro, sulla falsariga di quanto già avviene in alcune regioni. Qualcosa di analogo potrebbe essere previsto per la giustificazione delle assenze

${ }^{64}$ Cf. «Bozza d'intesa tra la Repubblica Italiana e l'Associazione Musulmani Italiani», in Quaderni di diritto e politica ecclesiastica 2 (1996), pp. 536-545; «Bozza d'intesa tra la Repubblica Italiana e la Comunità Religiosa Islamica», in Quaderni di diritto e politica ecclesiastica 2 (1998), pp. 567-575. La bozza d'intesa presentata dall'UCOII è disponibile sul sito internet <http://www. islam-ucoii.it/intesa.htm> (02 aprile 2003).

65 Tale analisi è confluita nel libro, a cura di Silvio Ferrari, Musulmani in Italia. La condizione giuridica delle comunità islamiche, Il Mulino, Bologna, 2000, qui più volte citato, sul quale si veda la recensione di Agostino Cilardo in Journal of Arabic and Islamic Studies 3 (2000), pp. 114-126. Per un'analisi del contenuto islamico delle bozze, si veda Cilardo, A., Il diritto islamico e il sistema giuridico italiano. 
scolastiche nelle festività religiose e nei «tempi di preghiera», attribuendo agli istituti scolastici il potere di regolare questa materia con disposizioni calibrate in relazione al numero delle richieste avanzate dagli alunni.

Infine vi sono materie che possono essere regolate solo per via di intesa. È il caso dell'insegnamento della religione islamica nelle scuole (con tutti i problemi connessi all'individuazione del personale insegnante) o dell'accesso al riparto dell'otto per mille dell'Irpef destinato alle confessioni religiose (con tutti i problemi connessi alla gestione di questa risorsa ed alla sua eventuale distribuzione fra le diverse comunità islamiche). In questi casi sarebbe preferibile-piuttosto che rimandare ogni soluzione ad una futura intesa generale-procedere per via d'intese specifiche e settoriali: da un lato, è probabilmente più facile che le diverse organizzazioni islamiche trovino un accordo su una questione particolare e circoscritta che sul complesso di questioni racchiuse in un'intesa generale; dall'altro, le intese particolari potrebbero essere il modo per avviare il processo di costruzione di una reale rappresentanza e per verificarne la solidità in vista di una successiva intesa generale.

Il principale ostacolo alla realizzazione di quest'ultima è dato proprio dalla mancanza di un unico organismo che rappresenti la comunità islamica italiana.

Sul terreno più propriamente giuridico è stata avanzata, per superare questa empasse, la proposta di stipulare molteplici intese con i diversi soggetti rappresentativi dell'islam italiano. Rispetto a questa ipotesi, che non pone problemi sul piano tecnico, va chiarito in via preliminare che le organizzazioni che rivendicano la rappresentanza dell'islam italiano non ricalcano le grandi divisioni religiose e giuridiche dell'islam: né quella tra sunniti e sciiti né quella tra le scuole hanafita, mālikita, šăficita e hanbalita. Un tale rilievo getta un'ombra sulla possibilità di considerare queste organizzazioni al pari delle diverse confessioni religiose cristiane e di replicare quindi, in riferimento alle prime, il modulo delle intese plurime che è stato applicato con le seconde. Le divisioni che separano le organizzazioni islamiche italiane non corrono in primo luogo lungo linee religiose, ma presentano motivazioni prevalentemente ideologiche e politiche: pare quindi legittimo chiedersi se concludere con esse più intese non significherebbe forzare la nozione di confessione religiosa e di conseguenza il testo dell'art. 8 Cost., che limita la possibilità di stipulare intese alle rappresentanze di confessioni religiose. Inoltre, secondo Cilardo "questa soluzione sarebbe inaccettabile per i musulmani in quanto essi credono nell'unità religiosa, anche se non più politica, della 
Umma islämiyya (comunità islamica). Sarebbe errato ritenere che il concetto di Umma abbia oggi un valore puramente teorico. Esso, invece, ha conservato tutto il suo carattere pregnante per la vita religiosa e civile dei musulmani in senso religioso e dottrinale. Essendo la Umma l'insieme di tutti i musulmani uniti dal vincolo di un'unica legge religiosa $\left(\check{s} a r \bar{r}^{c} a\right)$, sarebbe illogico pensare che ogni associazione islamica possa stipulare una propria intesa con lo Stato italiano, in quanto il contenuto dell'intesa, nelle linee essenziali, non potrebbe essere diverso per ogni gruppo di musulmani". 66

Alla luce di tutto questo, alla domanda che spesso ci si è posti negli ultimi tempi, ossia se riconoscere all'immigrato musulmano il diritto al mantenimento della propria identità comporti come conseguenza il riconoscimento del diritto di vivere secondo le proprie regole giuridicoreligiose, si può rispondere sostenendo che, "anche se l'identità confessionale esprime un valore costituzionalmente garantito, tale valore non è sovraordinato; vale a dire che, non solo l'ordine dello stato va distinto dall'ordine delle confessioni, ma che questa distinzione «presuppone la primazia (di fatto, ma anche assiologica) della sovranità dello stato, e dei valori che ne esprimono gli aspetti essenziali, nel suo ordine», primo fra tutti «il principio supremo di laicità»". ${ }^{67}$ In definitiva, uno Stato che si dichiari laico, anche se come quello italiano particolarmente attento alle esigenze religiose, non può consentire, e di fatto non consente, il prevalere del precetto religioso su quello civile. Quindi il musulmano, cittadino o straniero che sia, non può chiedere allo Stato il diritto di poter legittimamente applicare i propri precetti religiosi quando questi si pongono in conflitto con i diritti inviolabili dell'uomo che vengono riconosciuti e garantiti dalla costituzione. ${ }^{68}$

\footnotetext{
${ }^{66} \mathrm{Cf}$. Cilardo, A., recensione a: Ferrari, S. (a cura di), Musulmani in Italia, p. 119.

${ }^{67}$ Cf. Ivi, p. 117.

${ }^{68} \mathrm{Cf}$. Camassa Aurea, E., «L'immigrazione proveniente dai paesi islamici: conflitti ipotizzabili e soluzioni possibili», in Archivio giuridico 215,1 (1996), p. 48.
} 